\title{
Development of a Lever Mechanism for Loading a Suspended Conveyor
}

\author{
Ildar Kutlubaev ${ }^{1, *}$, Elena Macko $^{1}$, Igor Usov $^{1}$, and Olga Panfilova ${ }^{1}$ \\ ${ }^{1}$ Nosov Magnitogorsk State Technical University, 38, Lenin Ave., Magnitogorsk, Russia, 455000
}

\begin{abstract}
The issue of mechanization of hanging of long components of self-installing rock bolt (SIRB) type has been considered. The possibility of usage of slider-crank mechanism for these purposes has been proved. Linear dimensions of lever mechanism that meet the requirements defined by the applied motor and SIRB parameters have been determined on the ground of parametric synthesis. The performed force analysis has proved the application of fluid power drive.
\end{abstract}

\section{Introduction}

Movement of duplicate parts in coincidence with manufacturing operations including painting or coating is used in the production of a variety of goods. Usage of suspended conveyors is more preferable for such purposes as it will combine transport and manufacturing operations [1].

SIRB is actively used to fix working surfaces [2-4]. SIRB is a metal tube of an especial profile with diameter up to $50 \mathrm{~mm}$, length from 1,7 to $2,7 \mathrm{~m}$ and weight up to $8 \mathrm{~kg}$. Protection from aggressive environment impact is provided by means of especial coatings [5]. The technological line built and launched in OOO 'UralEnergoResusrs' is based on suspended conveyor. The most complex part of the technological process is loading of the suspended conveyor that is being performed by hand at present. The coating is applied simultaneously to 26 SIRB units fixed on one block. According to the cyclogram the time of hanging is 29 seconds. The pause between the following hanging operations is 60 seconds. A team of 5 workers is involved in a hanging operation.

SIRBs arrive at a loading section in registers horizontally layered on high from 600 to $1200 \mathrm{~mm}$ and in number up to 600 items. Hanging of each SIRB is performed by sequential operations:

a) Removing of SIRB from a register;

b) Moving to the conveyor for $2 \mathrm{~m}$;

c) Rotation of SIRB to $90^{\circ}$;

d) Lifting of SIRB in high from 0,5 to $1,5 \mathrm{~m}$;

e) Hanging of SIRB on a conveyor's hook.

The first four actions are performed by a team of workers. The last action is performed by another worker situated directly near the conveyor's hooks.

\footnotetext{
* Corresponding author: ptmr74@mail.ru
} 
Stopwatch study has shown that time and loading expenditures for execution of actions are divided into the following ratios: $\mathrm{a}-7 \%, \mathrm{~b}-18 \%, \mathrm{c}-38 \%, \mathrm{~d}-28 \%$, e- $9 \%$. The most labor intensive actions are rotation and lifting of SIRB.

\section{Problem statement}

Maximum number of actions should be mechanized in order to decrease labor contribution. Execution of the first action is performed with SIRBs that do not have their fixed position. The automated process of gripping and removing from register is possible only with usage of systems that provide: analysis of SIRB position, identification of the position and moving along the register. Actualization of operations requires to develop a robotic complex that reduces profitability of the production. This operation is not critical according to physical input. Execution of the second operation is connected with removing of SIRB and virtually is a part of it.

It is reasonable to develop a complex providing mechanization of operations $\mathrm{b}$ and $\mathrm{c}$. Limited working area around the conveyor makes it impossible to use two mechanisms. Both operations should be performed by one device.

\section{Consideration of engineering solution}

To perform $b$ and $c$ operations it is reasonable to use a lever mechanism. The simplest option is a four-link slider-crank mechanism (figure 1, a). It may be applied in a slidercrank mechanism variation (figure 1, b). In this case the sliding bar 3 is an input link and 1 is an output in. The structure of the mechanism is not changed here. Rotational movement of link 1 corresponds to the third operation and the additional lifting is provided in the presence of eccentricity that is how the fourth operation is performed.

Crank 1 is produced spread axially. It is used to fix lodgments on it to place the required number of SIRB. The length of lodgment $l$ exceeds the length of crank and comparable to SIRB length. because of substantial stroke of sliding bar 3 it is reasonable to use a pneumatic cylinder. Minimal working area of the whole facility is reached when it is placed under the links of lever mechanism (figure 1, d).

The main issue of slider-crank mechanism is maintenance of motion at the initial stage (figure $1, \mathrm{~b}$ ). When $\gamma$ values are close to $\pi$, the mechanism does not function. The most preferable value is $\gamma=\pi / 2$. In this case the loadings on the mechanism links are minimal. As far as crank 1 is approaching to the final position the angle $\gamma$ is decreasing (figure 1,c). The additional requirement is positioning of the drive within the lodgement dimensions (figure 1,d). 


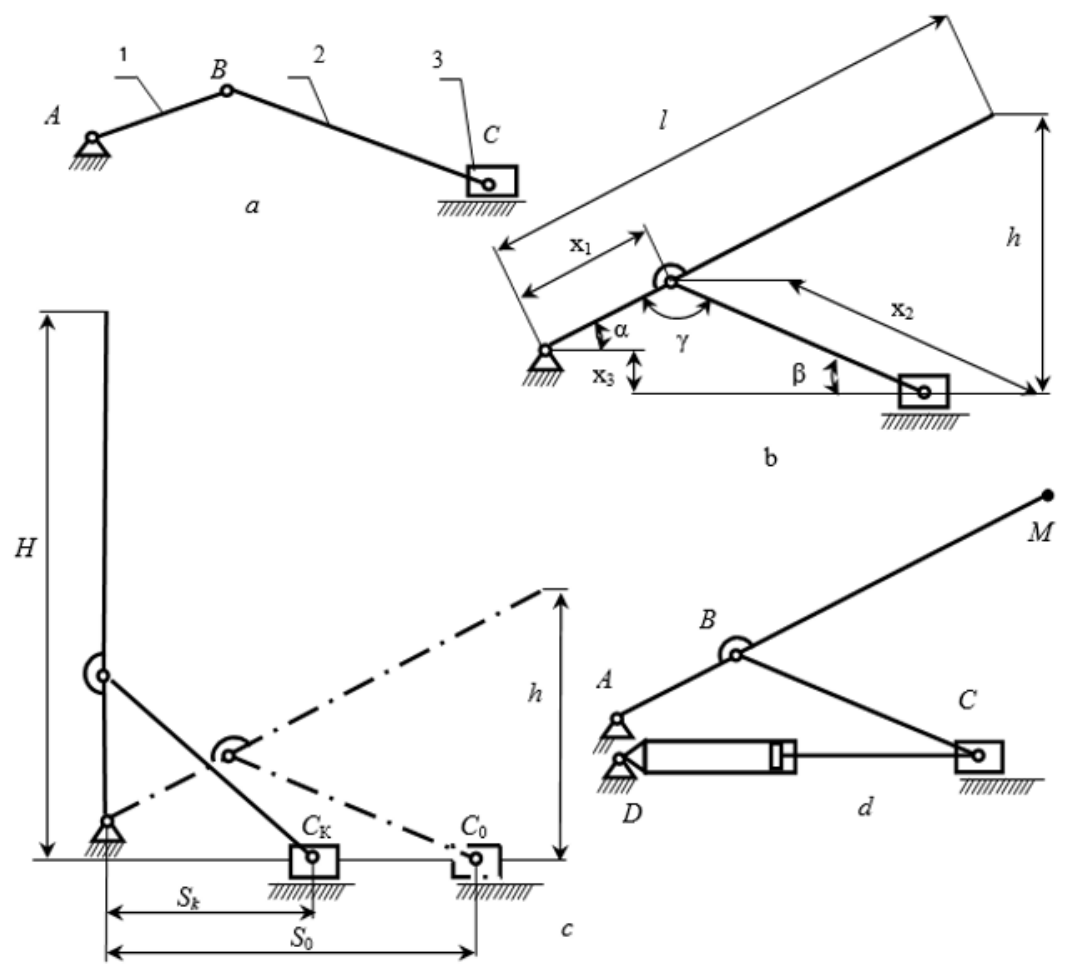

Fig. 1. Kinematic schemes: a) slider-crank mechanism, 1 - crank, 2 - bell crank, 3 - sliding bar; b) slider-crank mechanism to lift SIRB, c) end positions of slider-crank mechanism; d) slider-crank mechanism with pneumatic drive.

The priority is to determine linear dimensions of the slider-crank mechanism and the drive.

The initial parameters for parametric synthesis are the following:

$h$ - height determining the position of the end of lodgment in its initial location;

$l$ - length of lodgment.

The initial location of crank is determined by position of the end of lodgment at $h=900$ $\mathrm{mm}$ level. In this case minimal input is provided to perform $\mathrm{b}$ operations. The maximal length of lodgment $l$ must provide the stable position of SIRB with maximal length 2700 $\mathrm{mm}$. The condition is met when $l=1500 \mathrm{~mm}$.

\section{Parametric synthesis of lever mechanism}

The construction of lever mechanism is determined by linear dimensions (Figure 1, b):

$\mathrm{x}_{1}$ - length of crank

$\mathrm{x}_{2}$ - length of bell crank

$\mathrm{x}_{3}-$ eccentricity.

Working capacity of the construction is maintained when the following functional limitations are accomplished:

$$
\begin{aligned}
& g_{1}=\left(x_{2}-x_{1}\right)>0, \\
& g_{2}=l * \cos \alpha-s_{0}>0, \\
& g_{3}=s_{k} / \Delta s>k,
\end{aligned}
$$


where $\Delta \mathrm{s}$ is a slide stroke (cylinder piping)

$\Delta \mathrm{s}=\mathrm{s}_{0}-\mathrm{s}_{\mathrm{k}}$,

$\mathrm{s}_{\mathrm{k}}-$ dimension of pneumatic cylinder when $\Delta \mathrm{s}=0$;

$$
s_{k}=\left(x_{2}^{2}-\left(x_{1}+x_{3}\right)^{2}\right)^{0,5},
$$

$\mathrm{s}_{0}$ - initial position of sliding bar 3

$$
s_{0}=x_{1} * \cos \alpha+x_{2} * \cos \beta
$$

$\mathrm{k}$ - coefficient determined by pneumatic cylinder construction $\mathrm{k}=1,27 \ldots 1,3$.

Cranking capacity of lever mechanism is determined by condition (1). Condition (2) does not represent the exceedance of the drive dimension under the linear length of lever mechanism. Condition (3) represents executability of pneumatic drive.

Direct limitations $\mathrm{x}_{\mathrm{i}}>0,(\mathrm{i}=1 \ldots 3)$ should be implemented as well.

Optimality criterion is a function that represents minimal excursion of angle $\gamma$ from $\pi / 2$ value in initial position (Figure 1, b)

$$
F=\min (|\gamma|-\pi / 2)
$$

The value of angle $\gamma$ is expressed by projected parameters implicitly:

$$
\gamma=\pi-(\alpha+\beta)
$$

where $\alpha-$ a rotation angle of crank corresponding to the initial position of mechanism

$$
\alpha=\arcsin \left(\left(h-x_{3}\right) / l\right),
$$

$\beta$ - angle that determines the position of bell crank at initial position of lever mechanism

$$
\beta=\arcsin \left(\left(x_{1} * \sin \alpha+x_{3}\right) / x_{2}\right)
$$

According to optimization results it was found that $\mathrm{F}$ function takes a minimal value $37,3^{\circ}$ considering direct and functional limits and $\mathrm{x}_{1}=482 \mathrm{~mm}, \mathrm{x}_{2}=986 \mathrm{~mm}, \mathrm{x}_{3}=154$ $\mathrm{mm}$. In this case slide stroke is $\Delta \alpha=61^{\circ}$ and change of angle rotation of crank is $\Delta \alpha=61^{\circ}$.

\section{Force analysis of lever mechanism}

Strength prediction of the mechanism components and links and choice of pneumatic cylinder are based on the force analysis results. The original problem is to determine the put force to input link of mechanism and reactions in kinematic pairs.

The total weight of SIRB kit with length up to $2700 \mathrm{~mm}$ is $208 \mathrm{~kg}$. According to the preliminary consideration of receiving spars construction their weight is $174 \mathrm{~kg}$. The position of gravity center of loaded spar can be adequately taken at its end in point $\mathrm{M}$. The gross weight of link 1 is $\mathrm{m}=382 \mathrm{~kg}$ and in accordance the gravity force $\mathrm{G}=3747 \mathrm{~N}$. The weight of links 2 and 3 is negligibly small.

Inertial loads are applied to the mechanism links. Angular acceleration should be determined for their preliminary estimate. According to cyclogram of the conveyor performance, the time to lift SIRB is $t=6 \mathrm{sec}$. Based on symmetric diagram of velocity variation of the link, its average value is determined by:

$$
\dot{\alpha}=2 \Delta \alpha / t
$$


and angular acceleration:

$$
\ddot{\alpha}=2 \Delta \alpha / t 2 .
$$

With the adopted values of parameters of lever mechanism, the angular velocity is link 1 is $\dot{\alpha}=0,36 c^{-1}$ and angular acceleration is $\ddot{\alpha}=0,06 c^{-2}$.

Tangential acceleration of point of mass center is $a_{M}^{\tau}=l \ddot{\alpha}$. The normal one is $a_{M}^{n}=l \dot{\alpha}$.

After substitutions the absolute acceleration of point $\mathrm{M}$ will be $a_{M}=1,05 \mathrm{c}^{-2}$. Acceleration of point $\mathrm{M}$ is much smaller than gravity acceleration. In this regard the inertial loads in the calculation can be neglected.

Force P put to the slide stroke (cylinder piping) is determined by 'Zhukovsky lever' theorem. The velocity plan of slider-crank mechanism for initial position is presented in figure 2, a; 'Zhukovsky lever' is in figure 2, b.

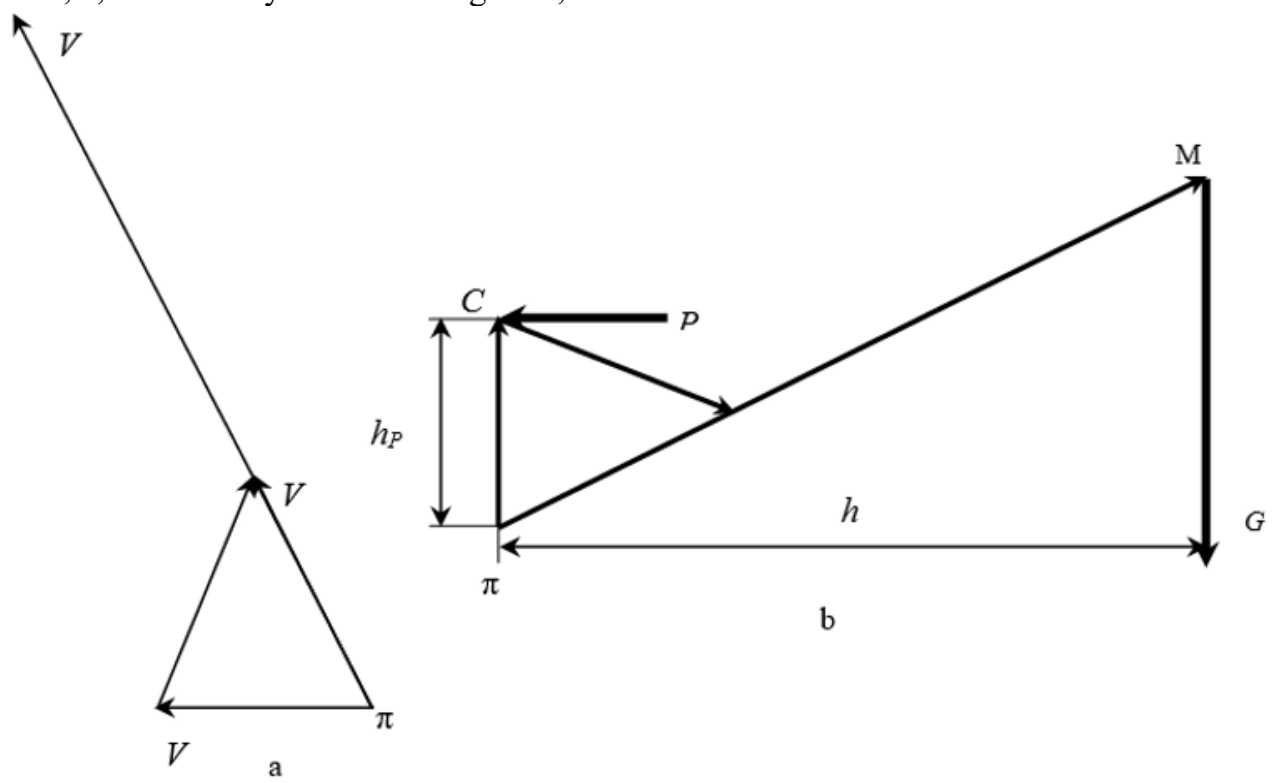

Fig. 2. Analysis of slider-crank mechanism: a - plan of possible velocities, b - 'Zhukovsky lever'

The correlation $r=h_{G} / h_{P}$ equals 3,3 due to 'Zhukovsky lever'. The correlation of the put force $\mathrm{P}$ to gravity force $\mathrm{G}$ equals $\mathrm{r}$ that is analogic as well. The put force equals

$$
P=r_{G}=3,3 * 3747=12366 \mathrm{H} .
$$

The required effort is made by pneumatic cylinder $1600 \mathrm{~mm}$ in functional diameter and at a pressure of $0,63 \mathrm{MPa}$.

\section{Conclusions}

Mechanization of hanging process of long components of SIRB type can be performed with usage of modified slider-crank mechanism.

The performed parametric synthesis of lever mechanism has made it possible to determine geometric dimensions that provide compact installing of the whole facility according to the suggested optimality criterion.

The lifting and rotation of SIRB block can be performed by pneumatic drive. 


\section{References}

1. N. P. Berlin, S. V. Smyshlenova, Complex mechanization and automization of loadingunloading operations, Gomel: Belarusian State University of Transport, 235 (2005)

2. A. A. Zubkov, A.V. Zubkov, I. M. Kutlubaev, V.V. Latkin, Improvement of friction rock bolt anchorage design and installation, Gornyi J, 5, 48-52 (2016)

3. Komurlu E., Kesimal A., and Colak U. Polyurea type thin sprayon liner coating to prevent rock bolt corrosion. Proc., 8th Asian Rock Mechanics Symp., Japanese Society for Rock Mechanics, Sapporo, Japan, 2014, pp. 1389-1397.

4. V. N. Kalmykov, A. A. Gogotin, A. N. Ivashov, Yu. D. Mambetova, The rationale behind the development sequence for a cluster of closely spaced copper-pyrite deposits using underground method, Vestnink of Nosov Magnitogorsk State Technical University, 15 (2), 21-27 (2017)

5. Zubkov A.A., Kutlubaev I.M., Leont'eva E.V. and Turkin I. S. Formation of Requirements of Reliable Operation of Frictional Fixation Rock Bolts IOP Conference Series: Earth and Environmental Science, Volume 272, Issue 2 IOP Conf. Series: Earth and Environmental Science 272 (2019) 022052 doi:10.1088/1755-1315/272/2/022052 